\title{
A Contrastive Study of Time as Space Metaphor in English and Chinese*
}

\author{
Cheng Chen \\ School of Foreign Languages, Zhejiang Gongshang University, China
}

\begin{abstract}
Time is an abstract concept which is invisible and intangible. The cognitive linguistics holds that the temporal concept is originally projected through the spatial concept. In the spatial temporal metaphor aspect, the traditional cognitions and theories are based on dynamic mode with facing the future: the time moving metaphor and the ego moving metaphor. Such cognition is generalized and not exact enough, unable to explain many English and Chinese temporal metaphors. Therefore, based on this problem, according to the cognition to the spatial position and the different visions of the observer, on the one hand a supplement is made to analyze the identifications and differences in English and Chinese temporal metaphors at the horizontal level with the static spatial-temporal mode in which the observer facing the past; on the other hand at the vertical level the spatial temporal metaphors are expounded with different observation points and the observer's states.
\end{abstract}

Index Terms - a contrastive study of English and Chinese, time, space metaphor

\section{INTRODUCTION}

Time is an abstract concept which is invisible and intangible. The cognitive linguistics holds that the temporal concept is originally projected through the spatial concept. Therefore, in comparative studies on English and Chinese temporal metaphors, the aspect with most importance is the 'spatial-temporal metaphor'. A great many papers are specialized to focus on this field. For example, Chen (2007) studies the similarities and differences of English and Chinese temporal metaphors on the basis of Lakoff's "time passing is motion" and analyzes it at both the horizontal level and the vertical level. Zhen (2009) researches two classical metaphorical ways-"time is moving" and "ego is moving", as well as the metaphors with the reference point of time and that of the observer. Nearly all of these researches followed Lakoff and Johnson's standpoint that "time passing is moving" and adapt it into the comparative study of English and Chinese temporal metaphors, but these spatial cognitions to the temporal map are not exact and clear enough, especially unable to explain a lot of Chinese linguistic phenomena. For example, most of the analyses explain the "ego-moving" spatial metaphor as a situation that time is an immovable and measurable space and the observer is movable from the past to the present forward the future, and the observer is facing the future. But this metaphorical type is too obscure and generalized, unable to explain clearly the spatial logic of language like the Chinese saying 前无古人, 后无来者(“unprecedented in history, unsurpassable for posterity"). There are also different opinions. For example, Zheng (2009) in A Study of Time as Space Metaphor in English and Chinese explains it with Núňez's (2006) time as the reference point metaphor. This metaphoric way sees the time passing as an axis with 'back' and 'front'. Here the earlier time is in the front and the later time is at the back. However, such an explanation ignores the existence of the observer and diverges from people's cognitive foundation in languages. Also it does not give out a clear time reference point. Moreover, it is confusing to include the "time is moving" and "ego is moving" in the observer as the reference point metaphor. In terms of the spatial vertical level, the analyses are simple and superficial, without elaborate expounds. For example, Chen (2007) in A Comparative Study of English and Chinese Temporal Spatial metaphors only explains the opposite situations with "unknown is up" and "known is down" in English and "unknown is down" and "known is up" in Chinese, losing the sight of similar cognitive situations in the two languages. In fact the circumstances are much more complicated. Therefore, this research would give out a rigorous perspective to study the spatial-temporal metaphor cognitions at both horizontal level and vertical level.

\section{Horizontal Level: ‘BACK’ AND ‘Front’ Temporal Location}

According to Lakoff's classical metaphorical cognition, there are two types: "time moving metaphor" and "ego moving metaphor". However, the division is not exact enough to analyze the spatial temporal concept exhaustively in the light of the cognition to spatial positions of the observer. For quite some time, in most cultures all over the world, modes for constructing temporal concepts are perceived from the spatial perspective, which are principally reflected as linear, cyclicity and helicity. Time is conceptualized as the general metaphor "Time Passing is Motion". In the linear mode, time is only in one dimension, moving from the past to the present and towards the future, or vice versa. In the

\footnotetext{
* This essay is supported by Project of Zhejiang Department of Education in 2013, China, Project Number: Y201327364.
} 
cyclicity mode, time is obviously in two dimensions, taking shape a circle, along which one can moves forward the past. In the helicity mode, time moves to three directions-'up' and 'down', 'back' and 'front', 'right' and 'left' at the same time in three dimensions. In temporal metaphors with spatial concepts in English and Chinese, the spatial orientations lay at two levels: the horizontal level and the vertical level.

1. Dynamic Spatial Temporal Metaphor Concept

According to the two basic types of temporal metaphor, which are 'time is the mover' (Time moving metaphor) and 'time is the container' (ego moving metaphor) (Lakoff, 1993, P.98), the linear movement at the spatial horizontal level produces the temporal cognition of 'back' and 'front'. Because 'back' and 'front' observe the principle of structural invariance while being projected from the spatial domain to the temporal domain, we would observe the cognitive tactics of 'back' and 'front' in spatial perspective naturally when we make a choice to the tactics of temporal cognition. Such cognition has two reference systems, which are subject reference and object reference, consequently the denotations of 'back' and 'front' of time are different. (Lakoff \& Johnson, 1999)

In terms of the property of unremittance of time, time is compared with mobile object at a finite speed permanently. The observer is comparatively static and the future is moving towards us, which refers 'time moving'. In this temporal spatial mode, the observer stands still and faces the coming time; time travels to the observer in the direction from the future to the past. There are back and front positions around the observer along the movement line. In order to understand the concept more explicitly, a scene is assumed in Figure1 that the observer is looking towards the future and watching the object coming up to him. At this moment, the observer would perceive that Object 2 is in front of Object 1; Object 4 is in front of Object 3 and the like; Object 5 is in front of Object 4. As the spatial concept in the source domain projects into the temporal concept in the target domain, Object 4 and Object 5 which represent the future are in front of Object 3 which represents the presence, and Object 3 is in front of Object 1 and Object 2 which represent the past.

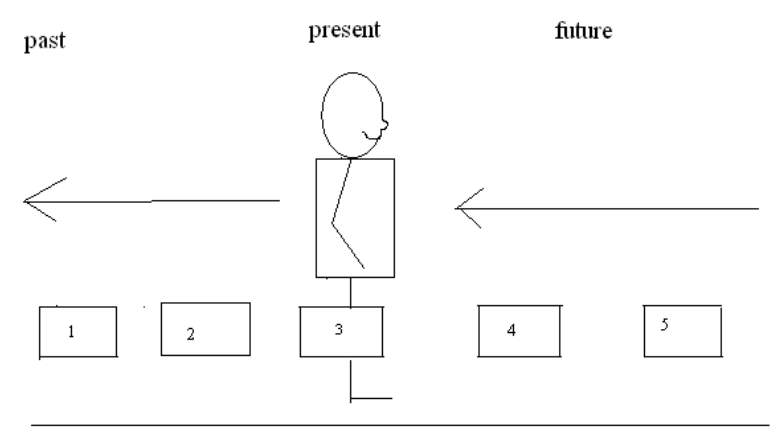

\author{
Notes: 1 refers to Object 1 \\ 2 refers to Object 2 \\ 3 refers to Object 3 \\ 4 refers to Object 4 \\ 5 refers to Object 5 \\ Figure 1 Time is Moving and the Observer is Static Facing the Future
}

The 'time moving metaphors' are found abundantly and commonly both in English and Chinese, including a great many verbs and prepositions. In English, words like come, ago, after, before and past, reflect the temporal concepts in the spatial domain. For instance, in the sentence The Christmas day is coming to us ., the time on the Christmas day in the future is conceptualized as the moving thing corporeal, which is traveling towards "us" but has not arrived yet. In the sentence Because although time passed by minute and second, the sentiments of loving you grow with each passing $\underline{\text { days. }}$, the observer "I" stands still and the time is passing over "me". Other similar examples with verbs conceiving temporal metaphors like The spring arrived earlier. and With the passing of time, everything changed. In Chinese, there are numerous words on time organized by the character 来(“come”), for example, 来世(“afterdeath”), 来生(“the other life") and 来日("days ahead"), which represent the time in the future. Here, 'the future time' is taken as things in the process of walking to the stationary observer. The character 往(“go") represents the past time, which is reflected in temporal words like 往事(“past events”), 以往(“in the past”) and 往年(“former years”). Common expressions like 考试 即将来临(“the examination is coming”), 时过境迁(“circumstances change with the passage of time”), 时来运转(“the luck has turned in one's favor"), 时间飞逝 (“time flies”), which embodies fully the characteristics that 'time is moving' and 'ego is static'.

Secondly, as every time quantum has its starting point and ending point, the observer can move during the limited time quantum, just like in a closed container. According to the spatial temporal metaphor, we take time as an immovable container. At this time the observer is moving from the position projected by the past time to the position projected by the present time till the position projected by the future time. In another word, it is the 'ego moving' metaphor(see 
Figure 2).

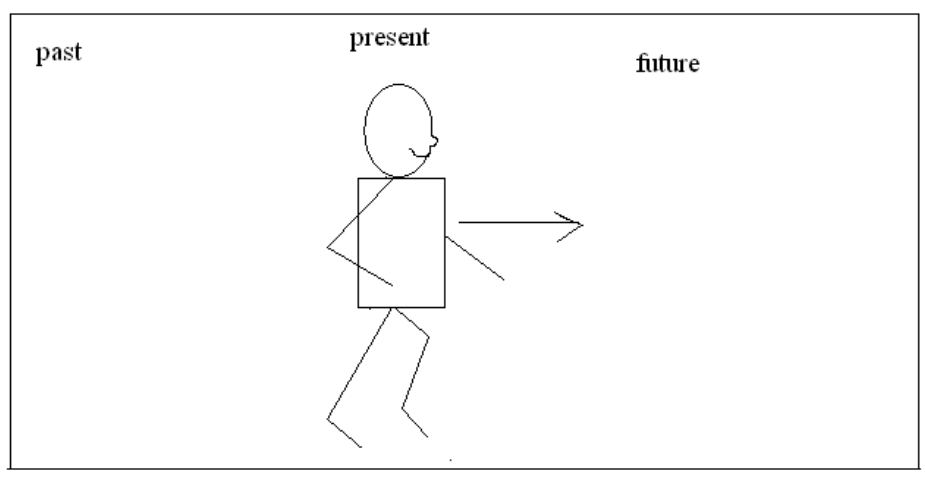

Figure 2 Time is a Stationary Container and the Observer is Moving Facing the Future

In the temporal concept of 'ego moving metaphor', time is seen as a fixed space and is conceptualized as a limited zone. The observer would finish walking the distance across this domain and go forward the further future. He is facing the future, crossing the space in the direction from the past to the present and to the future. Hence, 'ego moving metaphor' is also named as the 'time's landscape metaphor'.

In English there are plenty of 'ego moving metaphors' indicating 'future', such as get close, come up on and look back. For example, in the sentence We have left the gloomy yesterday and are walking towards the bright tomorrow. Here the time "yesterday" and "tomorrow" are motionless like the background and the observer "we" travels over the past time point towards the future time point till arriving. Taking another example, in the sentence We are coming up on the Thanksgiving Day. , "Thanksgiving Day" is a future time point in the place ahead the observer and the observer "we" is moving towards this time point.

Such similar expressions are ample in Chinese, too. For example, those daily used sayings like 历程 (“course") and 路程 (“journey") reflect that the observer walks over the corridors of time, which shows the state of "ego moving and time still'. For example, in the sentence 亚洲各国要将一个友好合作的关系带入一个新的纪元。(“The Ancient countries will bring a friendly cooperative relationship into a new era."), the observer "Asian countries" is active and will take the friendly relationship and advance towards the stationary "new era". Other frequently-used proverbs with the 'ego moving metaphors' in Chinese such as 人到中年 (“a person reaches middle age"), 过春节 (“spend Spring Festival").

2. Static Spatial Temporal Metaphor Concept

The static spatial-temporal metaphor concept that faces the past time can be represented as that both the observer and the time are in motionless state. The observer faces the past and turns back to the future. If the location of the observer is the present, this position point is in coincidence with the reference point. The two points can be also separated from each other with the specified reference time point. The point relatively near the observer is referred to the 'front' position and the point relatively far from the 'observer' is referred to the 'back' position, which can be shown in Figure 3.

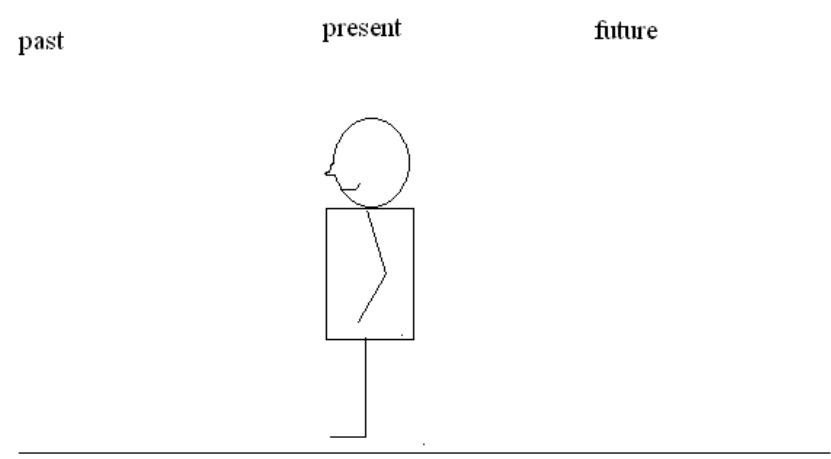

Figure 3 Time is Stationary and the Observer is Stationary Facing the Past

Therefore, 'front' is semantically transformed into 'the earlier time' and 'back' is semantically transformed into 'the 
later time'. It can be further divided into four sub-varieties.

First, the observer faces the past and against off the 'future' and the 'present' position corresponds with the temporal reference point. For example, in English, there are expressions like the day before, four years ago, three minutes ago; here after, after 3 years. In "four years ago", the observer stands on the position point 'present' and the 'target' time is in front of the observer with a distance of "four years'. "Here after" takes the position "here" as the reference point and represents the time at the back of the observer. In Chinese there are expressions like 两周前 (“two weeks age”), 以前 (“before”), 从前 (“once upon a time”), 前天 (“the day before yesterday”), 前年 (“the year before last year”), 日前 (“the other day”); 3 年后 (“three years later”), 以后 (“afterwards”), 今后 (“from now on”), 后患 (“future trouble”), 后市(“aftermarket”), 后事 (“arrangement for a funeral”). The word “日前” refers to the time earlier than today and “前天” refers to the time before the day before tomorrow; “后市” refers to the stock transaction in the time after today and “后事” refers to the business that has to be dealt with after death.

Second, the observer faces the past and sees two different time intervals. He defines the relatively farther time interval as the earlier period and the nearer time interval as the later period. The two time periods take each other as the time reference. The reference time point can be consistent with the observer's position, too. For example, in Chinese, there are expressions like 前任(“predecessor”), 前妻(“ex-wife”), 前科(“criminal record”), 前辈(“the senior”), 前生 (“prolife”); 后任(“successor”), 后劲(“aftereffect”), 后辈(“juniors”), 后记(“postscript”), 后生(“after born”). In Buddhism, “前生” refers to the body in the life in the previous existence ; “后劲” refers to the effect after drinking the wine, in which the time while drinking is seen as the earlier period and the time after that is seen as the later period. There are also a great many idioms with the similar cognition like 史无前例(“unprecedented in history”), 前车之鉴 (“warning taken from the overturned cart in front”), 革命老前辈(“a veteran of the revolution”), 前功尽弃 (“waste all the previous efforts”), 前所未有(“such as never previously existed”), 空前绝后 (“surpass the past and future”), 前无 古人,后无来者 (“A record that has never been approached and will never be approached again”); 后起之秀(“a promising youth”), 后继有人(“have qualified successors”), 后会有期(“There will be time for us to meet again”) in Chinese.

Third, the observer faces the past and sees a temporal reference point specified in language. The father point is taken as the front side and the nearer point is taken as the back side. The time reference is often in the past time and can be inferred to the future time. For example, in English, there are expressions like the day before yesterday, before I entered this school; before tomorrow's meeting; the day after tomorrow, after she finished work, after she comes back the next year. The phrase before I entered this school means the time when "I" had not but would enter the school. "The day when 'I' entered the school" is the reference time point, which is inconsistent with the present time, and the signified time is in front of the reference point as to the observer. In the phrase "after she finished work", the time that she finished work is specified as the reference point. Besides, there are words and affixes in English for representing this cognitive mode, like former, previous, ex-, pre-, post and so on.

In Chinese, there are expressions like 昨天午饭后(“after lunch yesterday”), 明天考试后(“after tomorrow's examination"). There are several representations that are derived from this cognitive mode, like 后工业化 ("post-industry") refers to a society no longer relying on heavy industry after the industrial revolution in $18^{\text {th }}$ century; 填迟 (“post-date") refers to a date fixed on a check that is later than the actual cashed date; 前科学(“prescience") and 前资本主义(“precaptalism”) which refers to the period that before the appearance of science and capitalism respectively;

Fourth, the observer faces the past without clear standing location. He sees a time interval and divides it into two parts. The father part from him is taken as the front side and the nearer part is taken as the back side. The two parts take references with each other. For example, in Chinese, there are expressions like 前半夜(“the first half of the night"), 前 半生(“the first half of one's life”), 战前(“prewar”), 前秦(“the Earlier Qin Regime”), which are corresponding with 后 半夜(“latter half of the night”), 后半生(“afterlife”), 战后(“afterwar”), 后秦(“the Later Qin Regime”). It is important to note that in Chinese, some temporal words with this metaphoric type are projected into the spatial directions on account of historic events. For instances, 南宋(“the Southern Song Dynasty”) and 北宋(“the Northern Song Dynasty”), 西汉(“the Western Han Dynasty”) and 东汉(“the Eastern Han Dynasty”). The movements of the capitals for the changes of regime in historical evolution lead to settlement of the directional cognition to the specified dynasties.

From the above analysis, we can see that the 'static spatial temporal metaphor concept' is both shared by in English and Chinese language, but it is more obvious in Chinese than in English, as we can see that certain metaphor modes are absent in English.

English and Chinese share the same metaphorical concept that 'time is space' both in dynamic cognitive mode and static cognitive mode. The events in the past are often settled and cannot be changed, so their temporal orders are referred by spatial metaphors. The future and the events in the future are indeterminate and mutable, so their temporal orders are referred by substantial metaphors. The spatial metaphor is the foundation of various temporal metaphors in English and Chinese languages for they have identical essence of spatial cognition and perception. Nevertheless, when reflected in specific language expressions, the two differ in thousands of ways. 
3. Distinctions of Temporal Metaphor Representations at Horizontal Level

First, at the horizontal static spatial level, Chinese is inclined to use the static spatial-temporal metaphor with facing the past to cognize the temporal order, while English is inclined to use specialized temporal words and ordinal numerals, or use the dynamic spatial-temporal cognition with facing to the future. For example, 今后的三年 is with the corresponding English version in the next three years; 后患 and 后福 are with the future trouble and future blessing respectively; 今后的生活 is with the English version the life ahead; 好戏还在后头呢 is with the interesting part is yet to come. 丑话说在前头 is with I would like to be strait firstly.

The static spatial-temporal metaphor with facing the past can be tinted with dynamic quality in Chinese, which cannot be found in English. For example, the Chinese temporal adverbial 十年以来 is with the corresponding English version in the last ten years in which the temporal concept is represented with static preposition. Similar expressions are like 时至今日 is with even today; 前年以来 is with since the year before last year. In the Chinese expression 从今往 后, it is in the static spatial- temporal metaphor with facing the past while in corresponding English translation from now on, it is in the dynamic spatial- temporal metaphor with facing the future.

In Chinese, “前' (“front”) and '后' (“back”) represent two cognitive modes that are 'the mode with facing the future' and 'the mode with facing the past'. However, the static cognitive mode with facing the past is more frequently employed in Chinese. “前” (“front”) and “后' ("back”) can be combined with a great many other characters to be used as nouns, adjectives, adverbs and prepositions. While in English, 'before' and 'after' only can be used as prepositions, but some affixes can refer to the temporal concepts as discussed above like precedent, ex-wife. For example, the Chinese versions 一周前, 四点后, 早餐后 are in correspondence with a week ago, before four, after breakfast.

While representing events that would happen in the future time, the dynamic 'ego moving' temporal metaphors are more likely to be adopted in English to form the metaphorical concept of spatial position 'future in the front' and 'past at the back' as the principal thing. In contrast, Chinese tend to employ the static metaphor system with concepts of 'past in the front' and 'future at the back' while representing temporal scope through spatial scope. For example, the English sentence: we are getting ahead of the part. has the corresponding Chinese translation that 我们要说到这一部分的后 面去了。. Another example is the English expression the last news, with the corresponding Chinese expression 最近的 新闻, instead of 最后的新闻. The sentence The story dates back to two hundred years. has the Chinese translation 故 事发生在两百年前.

Second, the preposition system for indicating spatial positions is very strong in English, but in Chinese it is relatively week. Quirk (1985) considers that when as prepositions of time, 'at', 'on' and 'in' are used in a way as equal as when they used as prepositions of place. Wang (2001) thinks the above discussed prepositions are indicating spatial positions-"spot", "surface" and "body". When they transform into representing time or abstract thoughts, they adhere to the projection regulation in the system of "spot", "surface" and "body".

For example, 'at' refers to the small spot in one dimension while representing position, and it refers to an extremely short time while representing time or time interval, like in expressions: at 6 o'clock, at the age of 10 and at a strike.

While 'on' refers to the location of the surface of the object in two dimensions while representing position, and it refers to a certain time point within a time interval or the close contact between two time points, for instance, $M y$ birthday is on June $12^{\text {th }}$.; On entering into the bedroom, he began to cry.; on the morning of March $25^{\text {th }}$.

The preposition 'in' refers to the location inside a cube in three dimensions while representing time, and refers to a period of time or a process while representing time, for example, in the morning, in the evening, in taking the bus.

There are many other temporal prepositions in English are transformed from spatial concepts. For example 'around' and 'about' signify the ambiguous meaning of 'around a certain range' temporally and spatially; 'between...and' and 'from...to' refer to the extension from the starting point to the finishing point. 'Over' refers to spanning in distance and time; 'through' and 'throughout' refer to running through the space and time (Zhang \& Ding, 2003). To be noticed, these prepositions are employed with clear distinguishment for indicating position and time, with each performing its own functions.

Contrarily, the Chinese prepositions of time, though are transformed from the spatial concepts, are not as accurate in semantics and as regulative in realization of lexical items as English prepositions of time, like “在' and ‘于’ . Therefore, the semantic motivation in English prepositions of time is much obvious than that in Chinese prepositions. This is also a proof for the common standpoint that English language is explicit while Chinese language is implicit.

Third, English verbs 'come' and 'go' are in correspondence with Chinese verbs 来 and 去. In Chinese, words for expressing time made up by 来 and 去 are too numerous to enumerate, such as 来年(“the next year”), 来日(“days ahead”), 将来(“future”); 去(“last year”), 去春(“last spring”). Furthermore, in Chinese words indicating dimensional orientation and words indicating temporal mapping have a fuzzy boundary in usage. The temporal meanings and spatial meanings co-conceive in most of the words, for example, “前' (“front") and “后' ("back") not only signify the spatial location, but also the temporal mapping. While in English, except a minority of phrases with 'coming' as modifiers like the coming weekend and the coming day, words 'come' and 'go' do not basically have such a function. There are words used especially for signifying special time, for instance, 'last', 'next' and 'past'. Although these words are extended from the spatial domain in meanings, they only can be used for temporal representations. This is because Chinese is a 
language at the morphonemic level and monosyllabic characters have a strong potential to build words. For example, '来' and '去' are able to combine with other characters to form various nouns and adverbs. However, 'come' and 'go' in English cannot be used as verbs.

\section{VerticAl LEVEL: 'UP’ AND 'DOWN' Temporal Location}

The analysis of the distinctions in English and Chinese temporal metaphors above is on representing spatial temporal metaphors at the horizontal level. There is also the metaphorical way that the vertical spatial movement is projected into the temporal mapping. In Chinese, the signifying rule is 'known for up' and 'unknown for down'. The metaphor value divides the space and time domain into halves. For example, the spatial word '上" ("up") indicates the earlier time and “下” (“down”) indicates the later time, such as 上半年 (“first half year”), 上辈子 (“past life”), 上午 (“forenoon”), 上周 (“last week”); 下半年 (“second half”), 下辈子 (“the next life”), 下午 (“afternoon”), 下周 (“next week”). Other temporal words like “高” (“high”), “低” (“low”), “头” (“head”), “尾” (“end”) which make up 高寿 (“longevity”), 低龄 (“young”), 年头 (“the beginning of the year”), 年尾 (“the end of the year”). This type of relation is reflected in Figure 4:

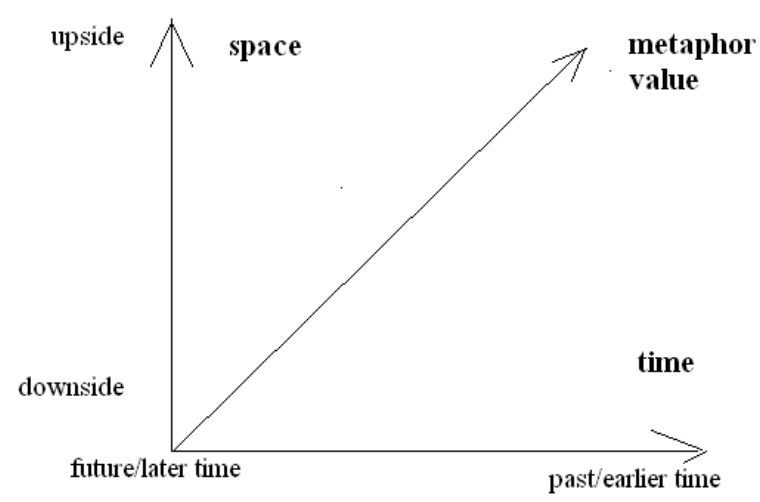

Figure 4 The Temporal Relation in the Lateral Order and Vertical Order in Chinese- 'Known for Up' and 'Unknown for Down'

It is supposed that the production of this kind of metaphor is related with the motion of sun, which means that the sun rises from the horizon in the morning, reaches the highest point at noon, so 上午 (“forenoon") represents the earlier time from that; when the sun begins to set in the afternoon till below the landline in the evening, the word 下午 (“afternoon”) comes out (Lan, 1999). The temporal expressions “上午” and “下午” show that Chinese projects the higher and lower positions in space into the temporal mapping, with the past time in the higher place and the future time in the lower place.

By comparison, in English, there are two situations.

First, the spatial position 'up' stands for the future and 'down' stands for the past with 'unknown for up' and 'known for down'. (see Figure 5) For example, Up to the end of last week, we had learned 8 units. ; Up to next month, we will have finished the project. . In Chinese, such a temporal metaphor concept can hardly be found.

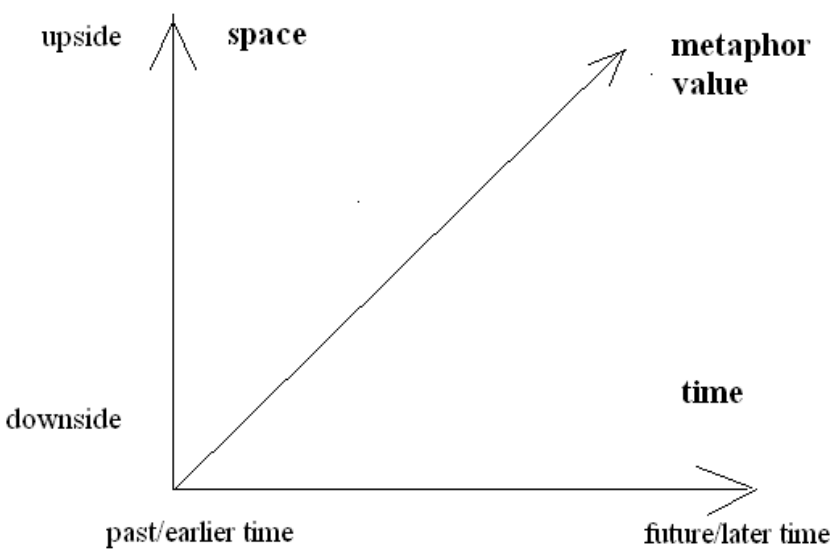

Figure5 The Temporal Relation in the Lateral Order and Vertical Order in EnglishI- 'Unknown for up' and 'Known for Down' 
Second, identical with the Chinese, there is a rare spatial-temporal metaphor at vertical level, in which the spatial position 'up' stands for the past and 'down' stands for the future. For example, word ascendant (先祖) for older people and descendant (后代) for younger people in family trees.

Situations analyzed above in English and Chinese are both in static aspect. As we can see that the static temporal metaphors on vertical space are comparatively rich in Chinese but infertile in English. However, there is another vertical spatial temporal mode in dynamic aspect in English, which is abundant in verb constructions with prepositions 'up' and 'down'. Here, the observer places himself over 'the future' and 'the past' (Radden \& Cuycken, 2003). When 'future' is in the downside, it is able to go upside to the position of the observer (see Figure 6).

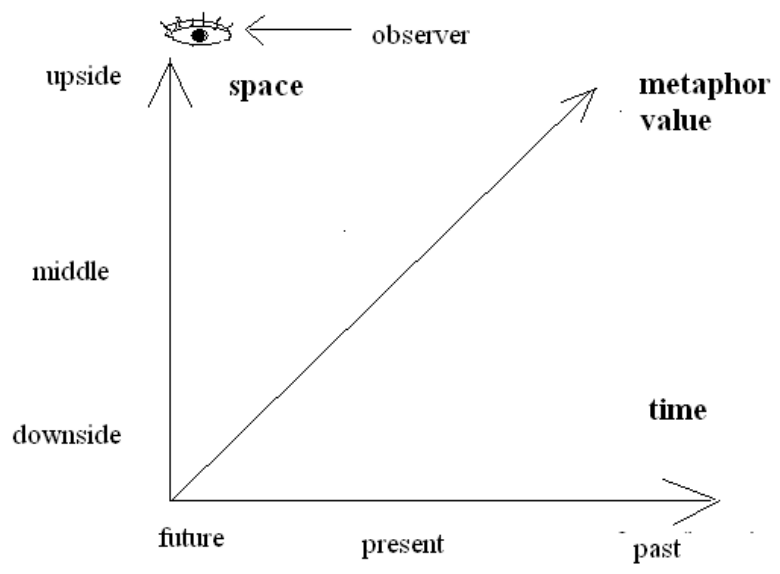

Figure6 The Temporal Relation in the Lateral Order and Vertical Order in EnglishII- 'Known for Up' and 'Unknown for Down'

For example, The Christmas day is coming up. (圣诞节即将到来) The future time point “Christmas day" is traveling from the downside to the upside from the visual angle of the observer. This story went down in a family history. (这个故 事在家族史中流传了下来) Here, “the story" is moving from the past time in the upside when the story came out towards the downside of the present time. Other example sentences like: The pearl has come down from my grandma. ; The festival can be traced up to 500 years ago. . However, prepositions related with concepts of 'up' and 'down' in English are in a very small quantity. The commonly used words are only up to and down to, but they are seldom utilized for indicating temporal concepts. Furthermore, there is expression-down through the ages, but there is no up through the past in English; and there is Descent of Man, but no Ascent of Apes. In Chinese, there are also several dynamic examples like 往上说(“recall the past"), 传下来(“hand down"), but they are not used as frequently as those in English.

To sum up, at the vertical level, dynamic spatial-temporal metaphors are much ampler than static spatial-temporal metaphors in English, while static spatial-temporal metaphors are much richer than dynamic spatial-temporal metaphors in Chinese. The temporal concepts expressed by horizontal spatial concepts play a dominant role in English language and the ways for signifying time through vertical dimensions are only a few to count.

\section{CONCLUSION}

Although 'spatial-temporal' metaphors are shared by both English and Chinese, but with the influence of language and culture, the metaphor representations show distinctions in directions and structures, which affect the cognitive way to the time or people. The above analysis has a comparison of the concepts and connotations of the English and Chinese temporal metaphors in dynamic spatial-temporal metaphor mode and static spatial-temporal metaphor mode. The result reflects that at the spatial horizontal level, English has a high frequency to use the dynamic spatial-temporal metaphors with facing the future, including the "ego moving metaphors" and "time moving metaphors", while in Chinese, the "time moving metaphors" are more commonly used than the "ego moving metaphors", but the static spatial-temporal metaphors with facing the past are more frequently used than the dynamic spatial-temporal metaphor mode. While at the vertical level, dynamic spatial-temporal metaphors are much ampler than static spatial-temporal metaphors in English, while static spatial-temporal metaphors are much richer than dynamic spatial-temporal metaphors in Chinese.

\section{REFERENCES}

[1] Chun Lan. (2005). A Study of Coganitive Linguistics and Metaphors. Peking: Foreign Language Teaching and Research Press.

[2] Jiafang Zhen. (2009). A Research on Spatial Metaphors of English and Chinese Temporal Concepts. Journal of Anhui University of Technology, 2, 86-88.

[3] Jianli Zhang \&Zhanping Ding. (2003). A Contrastive Study of Temporal Metaphors in English and Chinese Words. Foreign Languages and Their Teaching, 9, 31-34.

[4] Lakoff, G. (1993). The Contemporary Theory of Metaphor. Cambridge: Cambridge University Press.

[5] Lakoff, G. \& Johnson, M. (1999). Philosophy in the Flesh: The Embodied Mind and Its Challenge to Western Thought. New 
York: Basic Books.

[6] Núňez, R., Motz, B., \& Teuscher, U. (2006). Time after time: The Psychological Reality of the Ego-time-reference-point Distinction in Metaphorical Construals of Time. Metaphor and Symbol, 21, 133-146.

[7] Quirk, R. (1985). A Comprehensive Grammar of the English Language. London and New York: Longman.

[8] Radden, G. \& Cuycken, H. (2003). Motivation in Language. Philadelphia: J. Benjamins Pub. Co.

[9] Xiaoping Chen (2007). A Contrastive Study of English and Chinese Temporal Metaphors as Space. Journal of Changsha University, 6, 109-201.

[10] Yin Wang. (2001). Linguistic Theories and Language Teaching. Shanghai: Shanghai Foreign Language Education Press.

Cheng Chen is a researcher in the field of Foreign Languages and Applied Linguistics. She is an assistant at School of Foreign Languages, Zhejiang Gongshang University. Her main research interest is in languages and cultures, focusing on contrastive study of English and Chinese languages. 